\title{
Micro-genetic algorithm design of hybrid conventional waveguide and photonic crystal structures
}

\author{
J. Cai \\ S. Kim \\ Gregory P. Nordin \\ nordin@byu.edu \\ J. Jiang
}

Follow this and additional works at: https://scholarsarchive.byu.edu/facpub

Part of the Electrical and Computer Engineering Commons

\section{Original Publication Citation}

S. Kim, G. P. Nordin, J. Jiang, and J. Cai, "Micro-genetic algorithm design of hybrid conventional waveguide and photonic crystal structures," Opt. Eng., 43(9), pp. 2143-2149 (24)

\section{BYU ScholarsArchive Citation}

Cai, J.; Kim, S.; Nordin, Gregory P.; and Jiang, J., "Micro-genetic algorithm design of hybrid conventional waveguide and photonic crystal structures" (2003). Faculty Publications. 1024.

https://scholarsarchive. byu.edu/facpub/1024

This Peer-Reviewed Article is brought to you for free and open access by BYU ScholarsArchive. It has been accepted for inclusion in Faculty Publications by an authorized administrator of BYU ScholarsArchive. For more information, please contact ellen_amatangelo@byu.edu. 


\section{Microgenetic algorithm design of hybrid conventional waveguide and photonic crystal structures}

\author{
Seunghyun Kim \\ Gregory P. Nordin, MEMBER SPIE \\ Jianhua Jiang \\ Jingbo Cai, MEMBER SPIE \\ University of Alabama in Huntsville \\ Nano and Micro Devices Center \\ Huntsville, Alabama 35899 \\ E-mail: nordin@eng.uah.edu
}

\begin{abstract}
We previously proposed the hybrid integration of photonic crystals (PhCs) and conventional index-guided waveguides (CWGs) as a potentially attractive method of realizing compact waveguide elements for large-scale planar lightwave circuits (PLCs). We now examine 90-deg bends and beamsplitters in PhC/CWG structures in which the waveguide core has a high refractive index (3.25) and yet a low refractive index contrast $(1.54 \%)$ with the clad material. A PhC structure composed of a triangular or square array of air holes is placed at the intersection of input and output waveguides to obtain high efficiency 90 -deg bends. We find that diffraction from the boundary of the $\mathrm{PhC}$ region with CWG limits the optical efficiency of the bend. To overcome this we use a rigorous design tool based on a microgenetic algorithm $(\mu \mathrm{GA})$ and a finite difference time domain (FDTD) method to optimize the boundary layer to suppress the unwanted diffraction. We find that this approach yields improvements in the bend efficiency at a wavelength of $1.55 \mu \mathrm{m}$ from 56.2 to $92.5 \%$ (for a triangular PhC structure, TE polarization) and from 72.0 to $97.4 \%$ (square PhC structure, TM polarization). () 2004 Society of Photo-Optical Instrumentation Engineers. [DOI: 10.1117/1.1780546]
\end{abstract}

Subject terms: integrated optics; photonics; guided waves; optical devices; waveguides; photonic crystal; photonic integrated circuits; planar lightwave circuit.

Paper 030381 received Aug. 7, 2003; revised manuscript received Nov. 24, 2003; accepted for publication Mar. 2, 2004. This paper is a revision of a paper presented at the SPIE conference on Photonic Crystal Materials and Devices, Jan. 2003, San Jose, CA. The paper presented there appears (unrefereed) in SPIE Proceedings Vol. 5000.

\section{Introduction}

Realization of ultracompact planar lightwave circuits (PLCs) has been an active area of research ${ }^{1-6}$ for over 20 years because of the potential to dramatically decrease the cost of photonic systems while simultaneously increasing their functionality. We recently proposed a new approach based on the hybrid integration of conventional waveguides (CWGs) and small photonic crystal (PhC) regions. ${ }^{7}$ In this approach, CWGs are used for transport of light while $\mathrm{PhC}$ regions are introduced to dramatically reduce the size of waveguide devices. This preserves the traditional advantages of CWGs such as straightforward design for singlemode operation, low propagation loss, and low dispersion while advantageously using the attractive properties of PhCs.

In Ref. 7, we examined high-refractive-index $\mathrm{PhC}$ posts embedded in a conventional waveguide with a low refractive index core and a low refractive index contrast. In this paper, we examine the opposite case in which the $\mathrm{PhC}$ region is comprised of air holes in a CWG with a high refractive index core and a low refractive index contrast with the cladding material. A triangular lattice $\mathrm{PhC}$ (which has a wider photonic bandgap for TE polarization than a square lattice) is used for a 90-deg bend for TE polarized incident light (in-plane electric field) while a 90-deg bend for TM polarized light (out of plane electric field) is achieved with a square lattice $\mathrm{PhC}$ (which has a wider directional bandgap for TM polarization than a triangular lattice). We use 2-D numerical analysis based on the finite difference time domain (FDTD) method $^{8}$ to analyze such PhC/CWG structures. In the case of a 90-deg bend, we show that the periodicity of the PhC boundary in conjunction with the high index of the core and clad regions gives rise to diffraction that limits the maximum optical efficiency of the bend. We then show that this can be overcome by changing the first layer of the $\mathrm{PhC}$ region to suppress this diffraction. We design these modified structures with the aid of a rigorous design tool that we have developed, ${ }^{9,10}$ which consists of a microgenetic algorithm $(\mu \mathrm{GA})$ for optimization combined with a 2-D FDTD method for rigorous electromagnetic computation of candidate structure properties. Application of this design tool results in increases of the optical efficiencies of the bends from 56.2 to $92.5 \%$ for TE polarized light and from 72.0 to $97.4 \%$ for TM polarized light. We also show that rows of air holes can function as highefficiency 90-deg beamsplitters having total optical efficiencies of $99.4 \%$ for TM polarization (49.8/49.6 splitting ratio) and $98.8 \%$ for TE polarization (50.6/48.2 splitting ratio).

In Sec. 2, we first examine 90-deg bends with triangular lattice $\mathrm{PhC}$ for TE polarized light and show optimized results based on the $\mu \mathrm{GA}$. In Sec. 3 , we look at the equiva- 


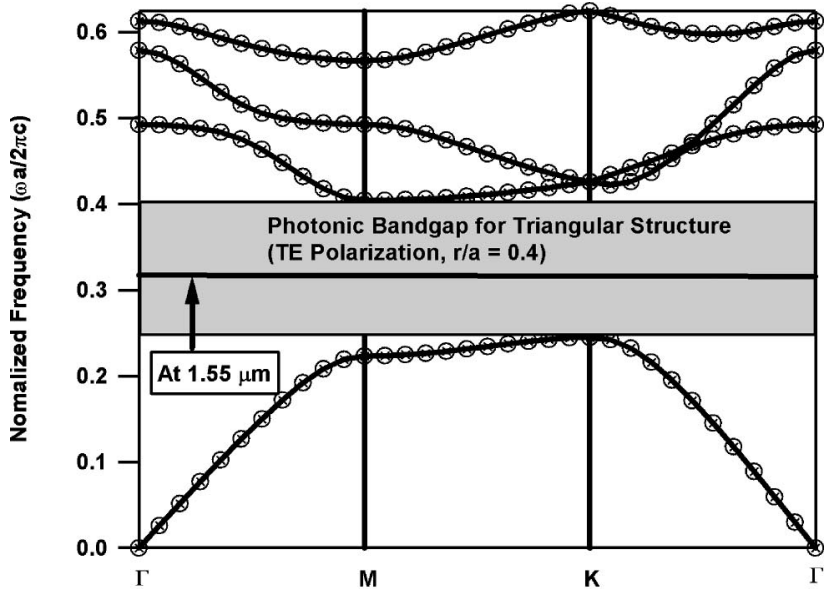

(a)

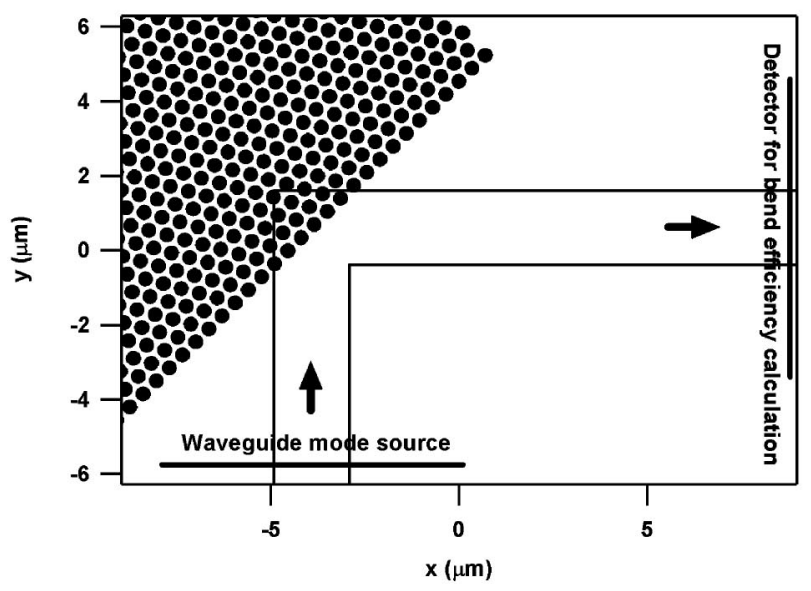

(b)

Fig. 1 (a) Dispersion relation of a $\mathrm{PhC}$ composed of a triangular air $(n=1.000)$ hole array in the waveguide material (assumed to be a quasi-homogeneous material with effective index of waveguide, $n$ $=3.239$ ) for $r / a=0.4$ and (b) geometry of the hybrid PhC/CWG structure.

lent case for a square lattice and TM polarized light. Highefficiency beamsplitters for TM and TE polarizations are then presented in Sec. 4.

\section{90-deg Bend-TE Polarization and Triangular PhC Lattice}

We first consider a hybrid $\mathrm{PhC} / \mathrm{CWG}$ 90-deg bend structure for TE polarized light. The core and clad refractive indices of the input and output waveguides for this case are 3.25 and $3.2(\Delta=1.54 \%)$ and their widths are $2 \mu \mathrm{m}$. As shown in Fig. 1(a), the dispersion relation of the triangular lattice $\mathrm{PhC}$ composed of air holes $(n=1.000)$ in the waveguide material, which is assumed to be quasi-homogeneous with the effective index of 3.239, for TE polarization reveals a photonic bandgap in the range of normalized frequency between $0.245\left(a / \lambda_{0}\right)$ and $0.404\left(a / \lambda_{0}\right)$ for $r / a=0.4(r$, radius of air holes of the $\mathrm{PhC} ; a$, period of the $\mathrm{PhC}$; and $\lambda_{0}$, wavelength in vacuum). We choose a normalized frequency of $0.319\left(a / \lambda_{0}\right)$, which is close to the middle of the photonic bandgap. For $\lambda_{0}=1.55 \mu \mathrm{m}$, the triangular lattice $\mathrm{PhC}$

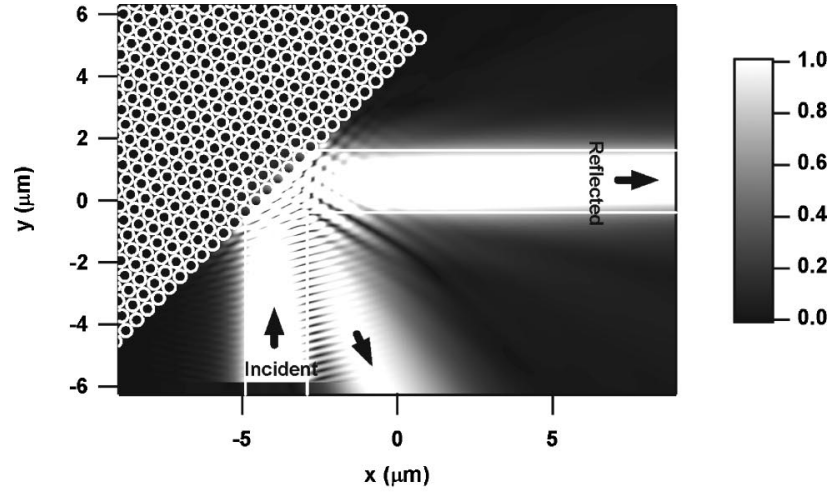

(a)

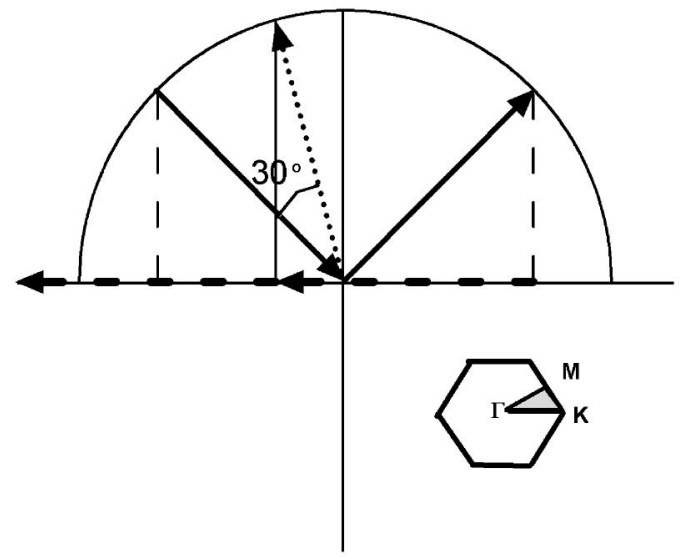

(b)

Fig. 2 (a) FDTD simulation result of magnitude squared time averaged magnetic field at $\lambda=1.55 \mu \mathrm{m}$ (Yee cell size, $15 \mathrm{~nm}$ ), and (b) wave vector diagram corresponding to the hybrid structure. Inset indicates the first Brillouin zone of the triangular lattice $\mathrm{PhC}$. The grating wave vector is calculated as $2 \pi / a$ (in which $a$ is the period of PhC lattice) and is oriented in the direction of the boundary of the $\mathrm{PhC}$.

has parameters $r=0.198 \mu \mathrm{m}$ and $a=0.495 \mu \mathrm{m}$. A hybrid 90-deg bend structure using this triangular lattice $\mathrm{PhC}$ is illustrated in Fig. 1(b). The incident waveguide mode source position and its propagation direction are indicated, and the length and position of a detector for calculating the bend efficiency (i.e., the ratio of detected power to the incident power) are also shown. The $\mathrm{PhC}$ is placed at the corner of the waveguides at 45-deg with respect to the input waveguide. The boundary layer of the $\mathrm{PhC}$ corresponds to the $\Gamma-\mathrm{K}$ direction of the triangular lattice.

The magnitude squared time averaged magnetic field (which, for the TE case, is the tangential field) for the hybrid structure at $\lambda=1.55 \mu \mathrm{m}$ is shown in Fig. 2(a). This result is calculated with a 2-D FDTD method with Berenger perfectly matched layer boundary conditions. ${ }^{11}$ From Fig. 2(a), we found that we cannot get high bend efficiency because a significant fraction of incident light is diffracted by the $\mathrm{PhC}$ and propagates in an unwanted direction. We examine the corresponding wave vector diagram shown in Fig. 2(b) to understand the behavior of this structure.

In Fig. 2(b), non- $\mathrm{PhC}$ and $\mathrm{PhC}$ regions are divided by the horizontal axis, which corresponds to the $\mathrm{PhC}$ boundary 


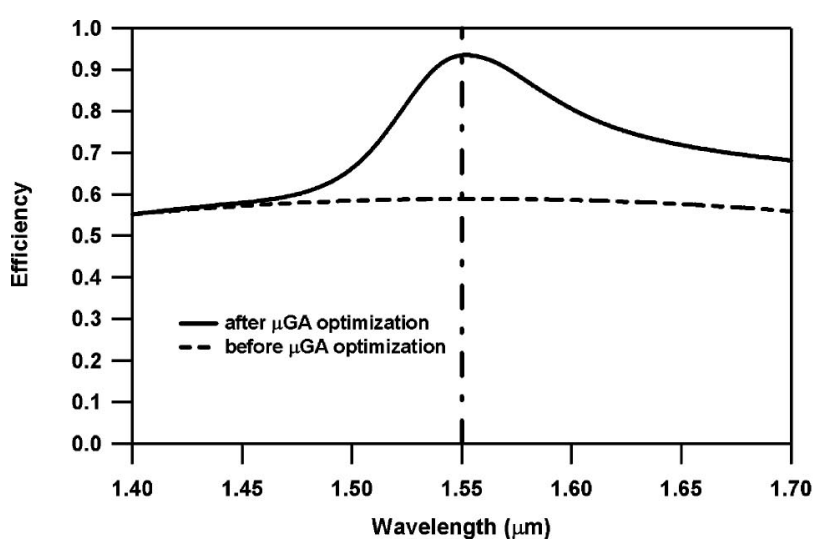

(a)

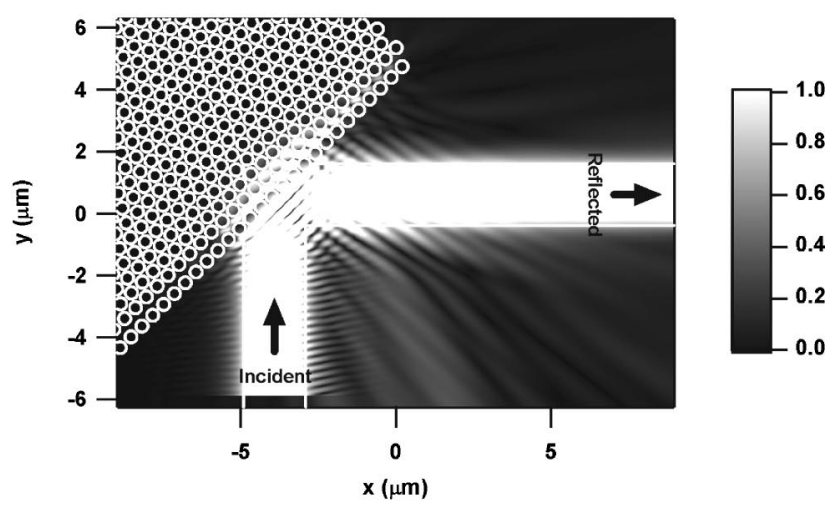

(b)

Fig. 3 (a) Bend efficiency comparison between the structures before and after optimization and (b) optimized hybrid structure and magnitude squared time averaged magnetic field for $\lambda=1.55 \mu \mathrm{m}$.

along the $\Gamma-\mathrm{K}$ direction of the triangular lattice. The semicircle in the upper half-space indicates the allowed wave vector in the CWG region. Since no possible mode exists in the $\mathrm{PhC}$, there is nothing in the lower half-space. Solid arrows show the incident and reflected light propagation directions while the grating vector associated with the periodicity of the $\mathrm{PhC}$ boundary is represented by dashed arrows and an allowed diffraction order direction is indicated as a dotted line. For 45-deg incidence of the waveguide mode on the PhC boundary, there is not only reflected light but also diffracted light propagating at a 30-deg angle with respect to the incident light. This agrees very well with FDTD simulation result in Fig. 2(a). It is clear that diffraction at the PhC boundary restricts the bend efficiency of this structure.

By modifying the boundary layer (i.e., the first layer) of the $\mathrm{PhC}$, we can manipulate the diffraction effect caused by the periodicity of the boundary. A $\mu \mathrm{GA}^{9,10}$ combined with 2-D FDTD is used to maximize the bend efficiency for $\lambda$ $=1.55 \mu \mathrm{m}$. The radius, period, and position of the air holes of the boundary layer are allowed to change to maximize the bend efficiency in the optimization process. Since there is a possible interaction between diffraction at the boundary and the allowed wave vectors in the $\mathrm{PhC}$, the size of the air hole for the $\mathrm{PhC}$ is also allowed to change while its period and position are fixed.

In Fig. 3(a), the bend efficiency as a function of wave-

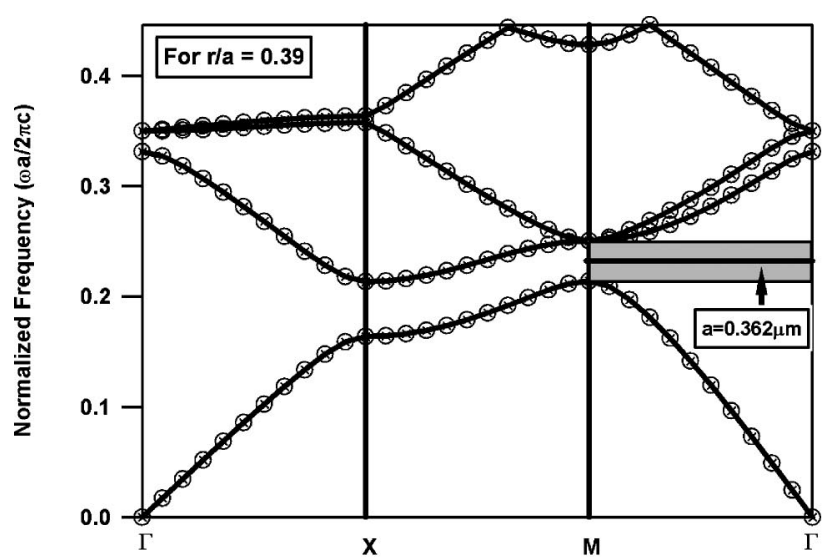

(a)

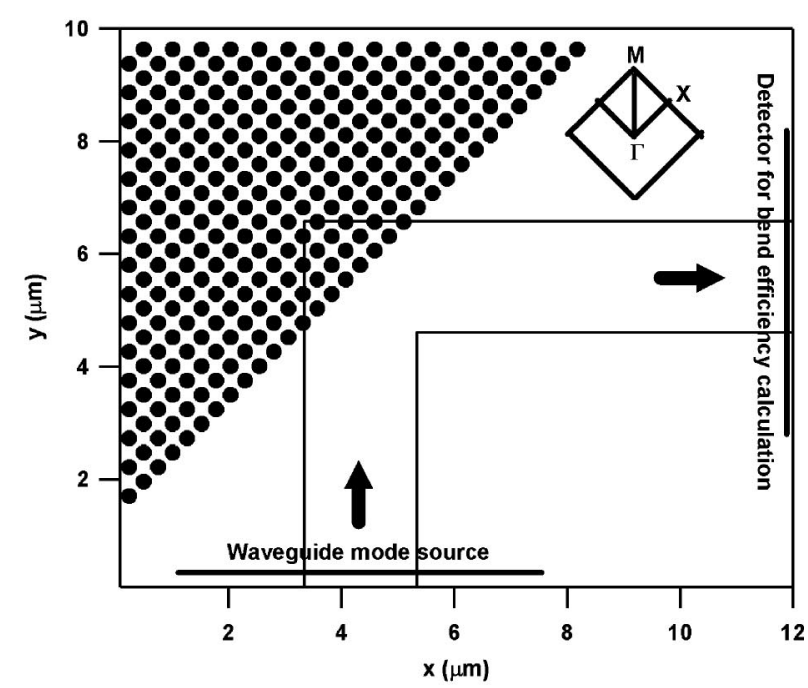

(b)

Fig. 4 (a) Band diagram for the PhC region of hybrid structure and (b) geometry of hybrid PhC/CWG bend for high-refractive-index waveguide and a PhC composed of a square array air hole lattice. A detector is used to calculate the bending efficiency. The diamond inset is the first Brillouin zone of the PhC.

length is compared before and after $\mu \mathrm{GA}$ optimization. Structure optimization with $\mu \mathrm{GA}$ clearly results in a significantly improved bend efficiency at the design wavelength of $1.55 \mu \mathrm{m}$. The optimized structure and the magnitude squared time averaged magnetic field for $\lambda=1.55 \mu \mathrm{m}$ is shown in Fig. 3(b). The radius and period of air holes for the boundary and the $\mathrm{PhC}$ of the optimized structure remains same as before $(r=0.198 \mu \mathrm{m}, a=0.495 \mu \mathrm{m})$. The only difference between the structures before and after optimization is the position of the boundary layer. The boundary layer is shifted toward the CWG region by $0.566 \mu \mathrm{m}$ as shown in Fig. 3(b), which in turn creates a small gap between the boundary layer and the $\mathrm{PhC}$ region. Destructive multiple beam interference for the diffracted light caused by this small gap suppresses the undesired diffraction order and increases the bend efficiency significantly from 56.2 to $92.5 \%$. 


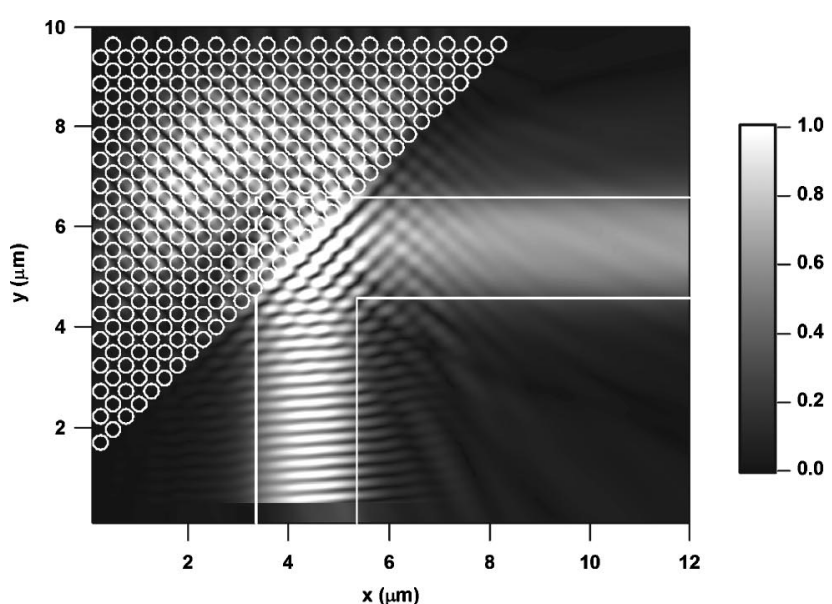

(a)

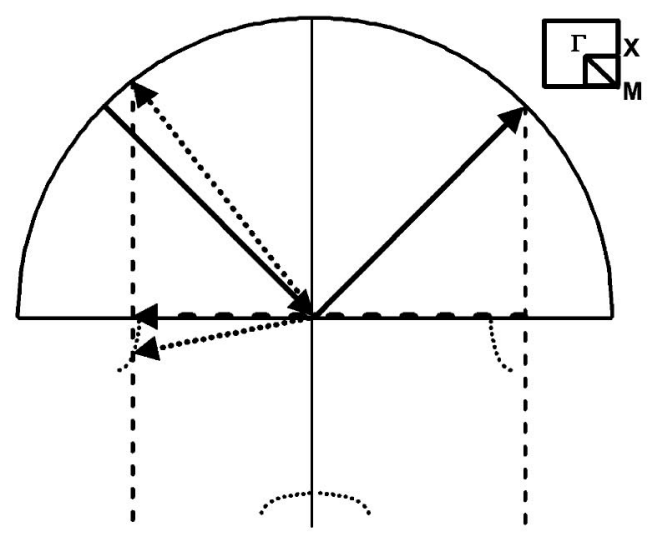

(b)

Fig. 5 (a) Magnitude squared time averaged electric field for TM polarized light for $\lambda=1.55 \mu \mathrm{m}$ and (b) corresponding wave vector diagram. Two solid arrows indicate the incident light and light reflected into the output waveguide. The dashed arrow indicates the grating vector for the periodicity of the first layer of the PhC. The dotted arrows denote the diffracted light. The inset shows the orientation of the first Brillouin zone with respect to the wavevector diagram. See Fig. 4(a) for how this relates to the bend structure orientation.

\section{90-deg Bend-TM Polarization and Square PhC Lattice}

A high-efficiency 90-deg bend can be realized for TM polarized light using a square $\mathrm{PhC}$ lattice and the same $\mathrm{CWG}$ as in Sec. 2. The band diagram of a PhC composed of a

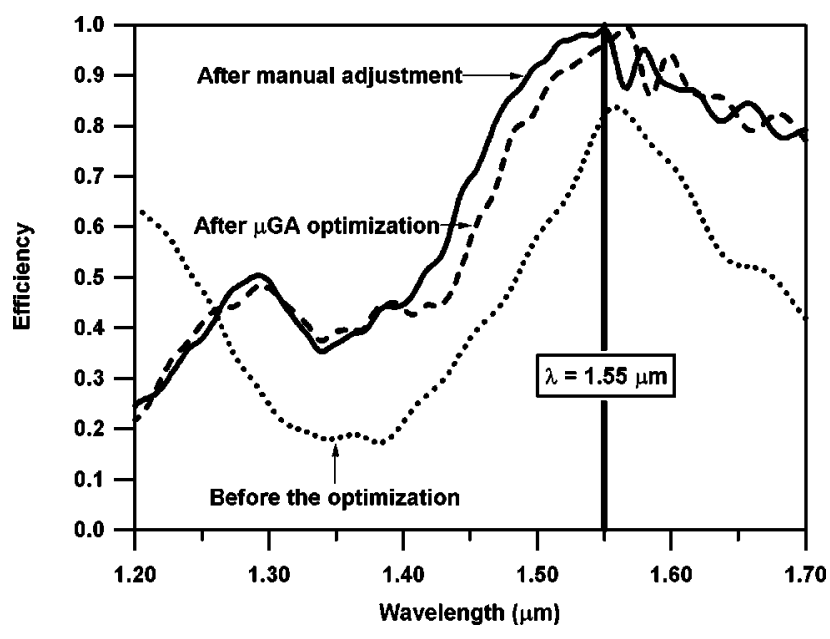

Fig. 6 Efficiencies as a function of wavelength for the initial structure (dotted line), the $\mu \mathrm{GA}$-optimized structure (dashed line), and the manually adjusted structure (solid line) with removal of the 75-nm minimum wall size limitation.

square air hole array in the $\mathrm{CWG}$ material (quasihomogeneous with $n_{\text {eff }}=3.239$ ) with $r / a=0.39$ is shown in Fig. 4(a). This square lattice $\mathrm{PhC}$ has a directional bandgap [ $\Gamma-\mathrm{M}$ lattice direction, normalized frequency range from $0.214\left(a / \lambda_{0}\right)$ to $\left.0.251\left(a / \lambda_{0}\right)\right]$ that is wide enough to create a high-efficiency 90-deg hybrid waveguide bend structure. We select a normalized frequency near the middle of the directional bandgap to obtain an initial design for the hybrid structure. The geometry of the initial design ( $r$ $=0.141 \mu \mathrm{m}, a=0.362 \mu \mathrm{m})$ is shown in Fig. 4(b).

Note that the boundary between the $\mathrm{PhC}$ and the CWGs corresponds to the $\Gamma$-X direction of the $\mathrm{PhC}$ lattice instead of the $\Gamma-\mathrm{M}$ direction for the low-index waveguide case reported in our previous paper. ${ }^{7}$ Use of the $\Gamma$-X direction as a boundary instead of the $\Gamma$-M direction presents a shorter period interface to the incident light, which decreases the number of possible propagating (i.e., nonevanescent) diffraction orders. This is a particularly important consideration for high-index CWGs in which the wavelength of light in the medium is substantially smaller than for lowindex CWGs.

In Fig. 5(a), the magnitude squared time averaged electric field (the tangential component of TM polarization) calculated by 2 -D FDTD for $\lambda=1.55 \mu \mathrm{m}$ is shown with the initial hybrid structure geometry. The bend efficiency is

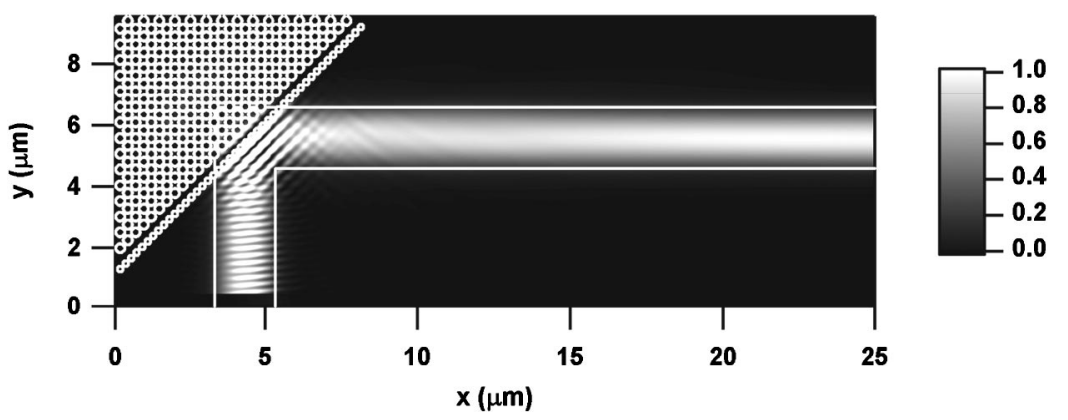

Fig. $7 \mu$ GA-optimized hybrid structure for high-index waveguide and simulation result for $\lambda=1.55 \mu \mathrm{m}$. 


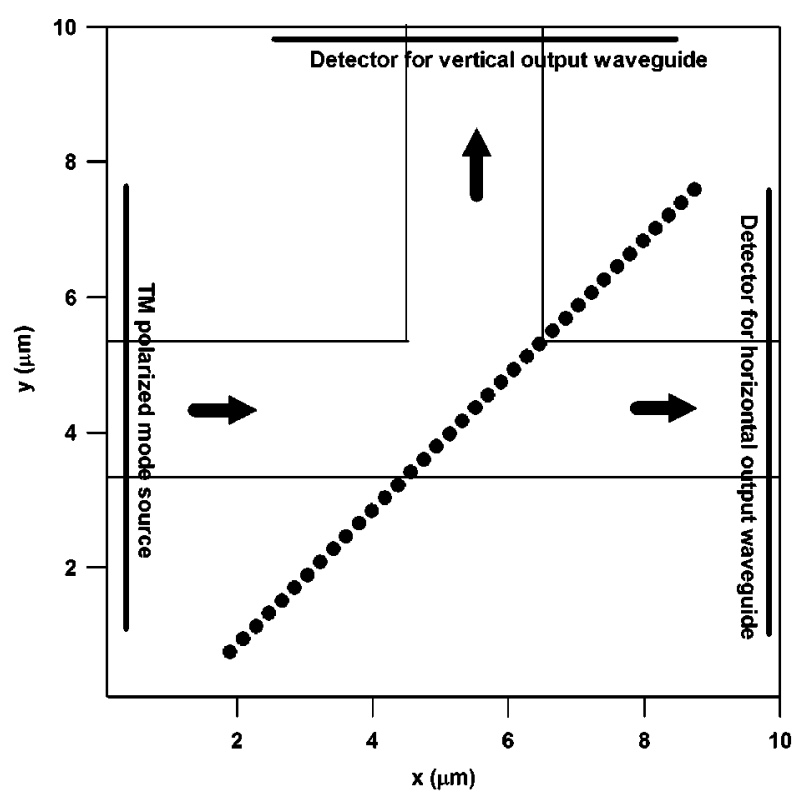

(a)

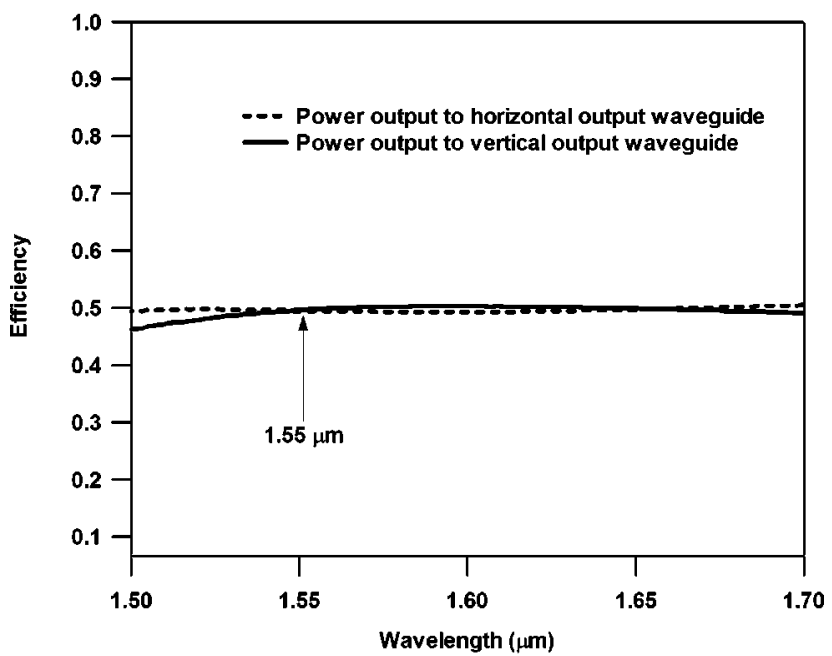

(b)

Fig. 8 (a) Geometry of high-efficiency beamsplitter for highrefractive-index and low-index-contrast waveguide (for TM polarization). Detector positions to calculate the efficiencies at the horizontal and vertical output waveguides are indicated. (b) The efficiencies at the horizontal and vertical output waveguides as a function of wavelength.

$72.0 \%$. A portion of the incident light is deflected backward toward the input waveguide while some propagates into the $\mathrm{PhC}$ region. The wave vector diagram for this structure is shown in Fig. 5(b).

The semicircle in the upper half-space of Fig. 5(b) indicates the allowed wave vectors in the waveguide region and the dotted curves in the lower half space denote the allowed modes in the $\mathrm{PhC}$. It is evident that there are two allowed diffraction orders from diffraction by the periodic $\mathrm{PhC}$ boundary. One of the orders propagates nearly back along the incident waveguide and the other couples to a $\mathrm{PhC}$ mode, as we notice in Fig. 5(a). The existence of these two diffraction orders limits the maximum bend efficiency that can be achieved.

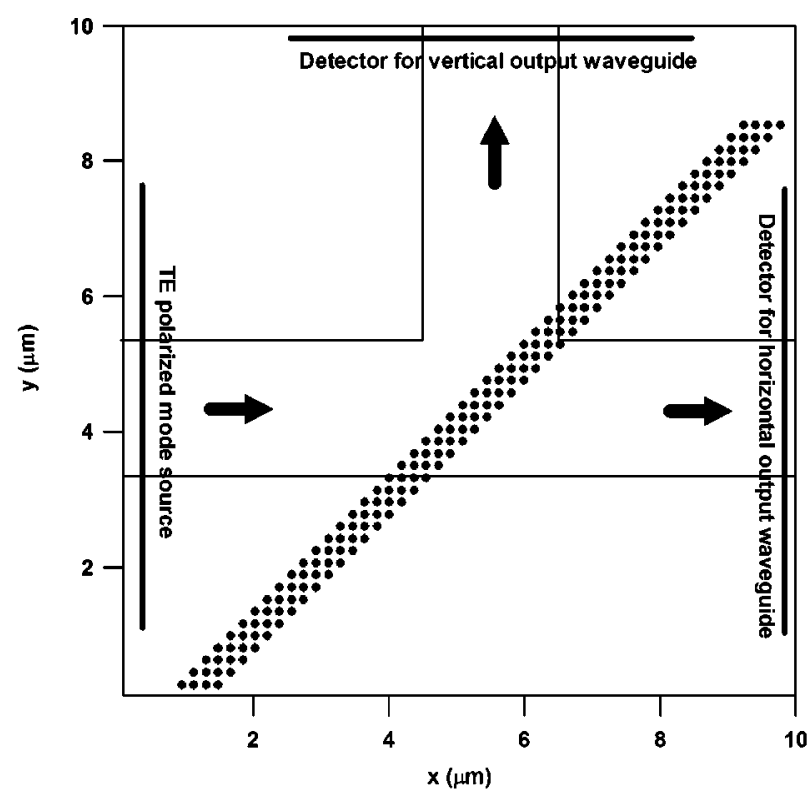

(a)

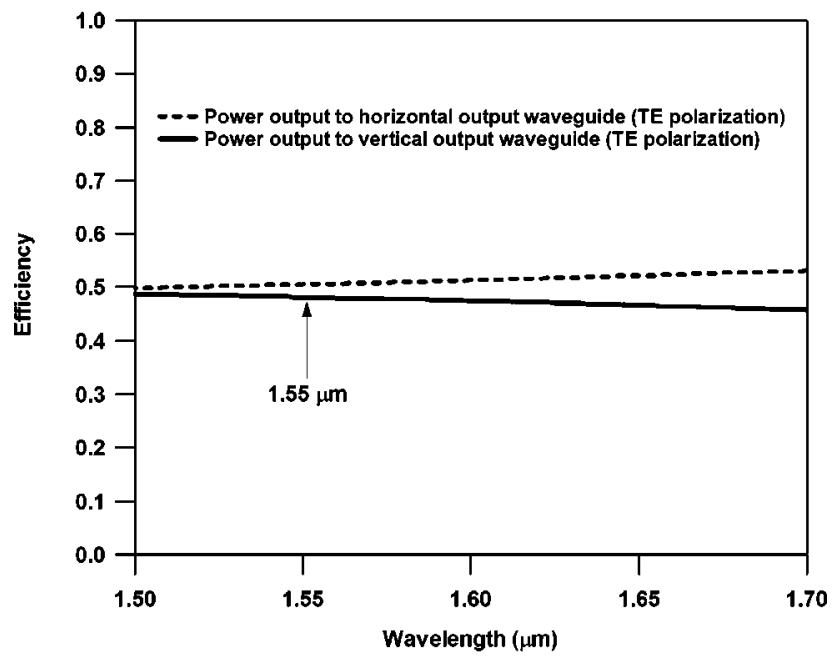

(b)

Fig. 9 (a) High-efficiency beamsplitter geometry for TE polarization with input waveguide mode source line and detectors for calculating efficiencies at both output waveguides and (b) spectral responses for the geometry shown in (a) at both horizontal and vertical output waveguides.

Optimization with $\mu \mathrm{GA}$ is again used to design a structure with improved bend efficiency. The parameters varied in the optimization are the radius and period of the boundary layer air holes, the position of the boundary layer, and the size of the air holes for the $\mathrm{PhC}$. The maximum size of the air holes in the bulk $\mathrm{PhC}$ region and the boundary layer is restricted such that the minimum allowable wall size (edge-to-edge separation of the adjacent two holes) is 75 $\mathrm{nm}$. Results for before and after $\mu \mathrm{GA}$ optimization are shown as the dotted and dashed lines, respectively, in Fig. 6 . The bend efficiency at $\lambda=1.55 \mu \mathrm{m}$ is improved significantly $(95.0 \%)$ and there is a range of wavelengths $(1.5133$ to $1.5773 \mu \mathrm{m}$ and 1.593 to $1.6067 \mu \mathrm{m}$ ) for which the bend efficiency is greater than $90 \%$. Although we optimized for only $\lambda=1.55 \mu \mathrm{m}$, a significant improvement of the bend 
efficiency was obtained over most of the wavelength range shown in Fig. 6. However, note that the peak bend efficiency is not at $1.55 \mu \mathrm{m}$. We found that this is caused by our restriction on the minimum 75-nm wall size, which limits the possible size of air holes of $\mathrm{PhC}$. The final optimized structure is obtained by increasing the size of air holes of the PhC manually, which in turn decreases the wall size, and the result is shown as the solid line in Fig. 6.

The geometry of the final structure and magnitude squared time averaged electric field for $\lambda=1.55 \mu \mathrm{m}$ are shown in Fig. 7. The air hole period of the $\mathrm{PhC}$ is $362 \mathrm{~nm}$, while the hole radius is $145 \mathrm{~nm}$. The boundary layer of holes has a period of $253.85 \mathrm{~nm}$ with a hole radius of 89.33 $\mathrm{nm}$. The position of the boundary layer is shifted 170.24 $\mathrm{nm}$ in the $-y$ direction, as seen in Fig. 7. Now the wall sizes of the $\mathrm{PhC}$ and the optimized boundary layer are 72 and $75.19 \mathrm{~nm}$, respectively. The bending efficiency at 1.55 $\mu \mathrm{m}$ is improved to $97.43 \%$.

\section{High-Efficiency Beamsplitters}

As illustrated in Fig. 8(a), a 90-deg beamsplitter can be formed with a row of air holes. The spectral response for TM polarized light and a one-layer air hole array with a $268.7 \mathrm{~nm}$ period and a $105 \mathrm{~nm}$ air hole radius are shown in Fig. 8 (b). At $\lambda=1.55 \mu \mathrm{m}, 49.8 \%$ of incident light is directed into the horizontal output waveguide, while $49.6 \%$ is coupled into the vertical output waveguide (for a total efficiency of $99.4 \%$ ).

Note that the spectral response of this is quite broad. This high-efficiency beamsplitter is essentially a subwavelength diffraction grating that generates no propagating diffraction orders beyond the transmitted and reflected zero orders.

We can alternatively create a beamsplitter for TE polarized light with a four-row air hole array, as shown in Fig. 9(a) (radius $=64 \mathrm{~nm}$, period $=255 \mathrm{~nm}$, row separation $=180 \mathrm{~nm})$. The spectral response of this structure is shown in Fig. 9(b). At $\lambda=1.55 \mu \mathrm{m}, 50.6$ and $48.2 \%$ of the incident light are directed into the horizontal and vertical output waveguides, respectively (98.8\% total efficiency).

\section{Summary}

Hybrid PhC/CWG bends in high-index, low-index-contrast waveguide material systems require careful design to avoid efficiency-limiting diffraction from the periodic $\mathrm{PhC}$ boundary. Our rigorous $\mu \mathrm{GA} / \mathrm{FDTD}$ design tool was shown to be effective in designing high-efficiency 90-deg bends by changing the properties of the boundary layer to suppress unwanted diffraction. Further studies are necessary to explore other device implementations, out-of-plane scattering loss, and the effects of fabrication tolerances.

High-efficiency beamsplitters for TM and TE polarizations using the given waveguide structure are designed by simply adding rows of 45-deg-tilted air hole array. Wide wavelength ranges of high-efficiency splitting are achieved for both TM and TE polarization.

\section{Acknowledgment}

This work was supported in part by the Defense Advanced Research Projects Agency (DARPA) Grant No. N6600101-1-8938 and National Science Foundation Grant No. EPS-0091853.

\section{References}

1. P. Buchmann and H. Kaufmann, "GaAs single-mode rib waveguides with reactive ion-etched totally reflecting corner mirros," J. Lightwave Technol. LT-3(4), 785-788 (1985).

2. R. L. Espinola, R. U. Ahmad, F. Pizzuto, M. J. Steel, and R. M. Osgood, "A study of high-index-contrast 90 degree waveguide bend structures," Opt. Express 8(9), 517-528 (2001), http:// www.opticsexpress.org/abstract.cfm?URI=OPEX-8-9-517.

3. M. Popovic, K. Wada, S. Akiyama, H. A. Haus, and J. Michel, "Air trenches for sharp silica waveguide bends," J. Lightwave Technol. 20(9), 1762-1772 (2002).

4. S. Lardenois, D. Pascal, L. Vivien, E. Cassan, and S. Laval, "Lowloss submicrometer silicon-on-insulator rib waveguides and corner mirrors," Opt. Lett. 28(13), 1150-1152 (2003).

5. J. D. Joannopoulos, R. D. Meade, and J. N. Winn, Photonic Crystals: Molding the Flow of Light, Princeton University Press, Princeton, NJ (1995).

6. C. Chen, A. Sharkawy, D. M. Pustai, S. Shi, and D. W. Prather, "Optimizing bending efficiency of self-collimated beams in non-channel planar photonic crystal waveguides," Opt. Express 11, 3153-3159 (2003), http://www.opticsexpress.org/abstract.cfm?URI=OPEX-1123-3153.

7. G. P. Nordin, S. Kim, J. Cai, and J. Jiang, "Hybrid integration of conventional waveguide and photonic crystal structures," Opt. Express 10(23), 1334-1341 (2002) http://www.opticsexpress.org/ abstract.cfm?URI=OPEX-10-23-1334.

8. A. Taflove, Computational Electrodynamics: The Finite-Difference Time-Domain Method, Artech House, Boston (1995).

9. J. Jiang and G. P. Nordin, "A rigorous unidirectional method for designing finite aperture diffractive optical elements," Opt. Express 7(6), 237-242 (2000) http://www.opticsexpress.org/abstract.cfm?URI $=$ OPEX-7-6-237.

10. J. Jiang, J. Cai, G. P. Nordin, and L. Li, "Parallel microgenetic algorithm design for photonic crystal and waveguide structures," Opt Lett. 28(23), 2381-2383 (2003).

11. J. P. Berenger, "A perfectly matched layer for the absorption of electromagnetic waves," J. Comput. Phys. 114(2), 185-200 (1994).

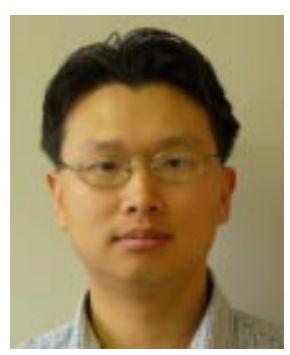

Seunghyun Kim received his BS degree in physics from the University of Suwon, Republic of Korea, in 1998 and his PhD degree in optical science and engineering from the University of Alabama, Huntsville in 2004 . He is currently a postdoctoral research associate with the Nano and Micro Devices Center of the University of Alabama, Huntsville. His research is focused on designs and fabrications of integrated planar photonic devices.

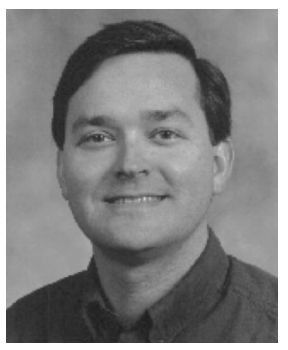

Gregory P. Nordin is the director of the Nano and Micro Devices Center (NMDC) and a Professor in the Department of Electrical and Computer Engineering at the University of Alabama in Huntsville (UAH) $\mathrm{He}$ received his $\mathrm{PhD}$ degree from the University of Southern California in 1992. His current research activities include photonic devices and high density planar lightwave circuit integration, nano and micro fabrication, MEMS and photonics-based sensors, and microfluidic devices. 


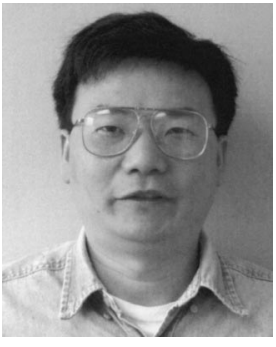

Jianhua Jiang is a research scientist with the Nano and Micro Devices Center (NMDI), the University of Alabama in Huntsville (UAH). He received his undergraduate degree in physics and his MS degree in optics from Tsinghua University, Beijing, China, in 1993 and 1996, respectively, and his $\mathrm{PhD}$ degree in optical science and engineering from the University of Alabama, Huntsville, in 2000. His research interests are rigorous grating theory and numerical modeling methods for photonic devices.

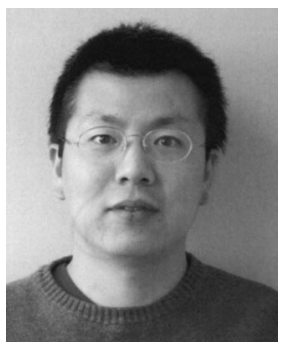

Jingbo Cai graduated from the Chongqing University, China, in 1991. From 1991 to 1996, he was an optical engineer with the Yunnan Optical Instrument Factory, China. He received his MSc degree in optical science from the Rose-Hulman Institute of Technology, in Terra Haute, Indiana, United States, in 1999, and is working toward his $\mathrm{PhD}$ degree in the optical science and engineering program at the University of Alabama, Huntsville. His current research interests include electromagnetic modeling and computer optimization of integrated optical components. 